\title{
Teknokultura
}

ISSNe: 1549-2230

http://dx.doi.org/10.5209/TEKN.57527

\section{Recordando el futuro, imaginando el pasado. La creación de escenarios como ejercicio ontológico ${ }^{1}$}

\author{
Mariana Ioana Gavris²; Francisco Tirado ${ }^{3}$
}

Recibido: 8 de octubre de 2017 / Revisado: 6 de diciembre de 2017 / Aceptado: 28 de diciembre de 2017. Open peer reviews

Resumen. Acercarse desde las lecturas de biopolíticas clásicas, para observar discrepancias que plantea la escalada del uso de nuevas tecnologías digitales en la epidemiología, permite identificar mecanismos para analizar cómo surge la permeabilidad de la membrana de lo biótico. Las propuestas basadas en la noción de "escenarios" sugieren nuevas articulaciones de sujetos y tecnologías. La transposición de los escenarios de salud pública global (como diseños estratégicos) — diseñados en las últimas décadasen los moldes digitales (epidemiología digital) supone cuestionarnos sobre la emergencia de nuevas ontologías sobre la vida. Desde los estudios de ciencia y tecnología (más específicamente de la teoría Actor-Red), se propone en el presente artículo la necesidad de replantear las lecturas tecnocientíficas a partir de tres ejes: a. bios traspuesto en datos; b. heterotopías en salud entre virtual-real; y c. los nuevos actantes que articulan sujetos y subjetividades. El análisis se plantea a partir de la triangulación de diferentes fuentes (escenarios y artículos periodísticos vinculados epidemiología; entrevistas personales y grupos focales) para observar cómo se articulan los mecanismos principales de la nueva ontología en salud, donde se reconfiguran las relaciones en la conceptualización de las políticas de salud global. Palabras clave: biopolítica; humanidades digitales; salud global.

\section{[en] New ontological exercises on life: scenarios in digital epidemiology}

\begin{abstract}
Approached from the classical biopolitical perspective, observations of discrepancies proposed by the escalated use of new digital technologies in epidemiology, allows us to identify particular mechanisms to analyze how the membrane of the biotic is being permeated. Proposals based on the notion of "scenarios" give rise to conceptual transformations that (will) involve new links between subjects and technologies. The transposition of the global public health scenarios (such as strategic designs) — designed in recent decades — into digital molds (digital epidemiology), brought us to inquire on emergence of new ontologies about life. Based on science and technology studies (more precisely, Actor-Network theory), the present article proposes the need to reconsider the techno scientific readings grounded in three main axes: a. bios transposed into data; b. heterotopies in health between virtual-real; and c. the new actors that articulate subjects and subjectivities. These analyses are based on the triangulation of different sources (scenarios and journalistic articles linked to epidemiology, personal interviews and focus groups) with an aim of observing the main articulation mechanisms of new ontology in health, which are reconfiguring the conceptual definition of global health policies.
\end{abstract}

1 Esta investigación se ha realizado en el marco del programa de doctorado Persona i Societat en el Món Contemporani, del Departamento de Psicología Social de la Universitat Autónoma de Barcelona; y forma parte de proyecto de investigación Salud y tecnociencia. La participación ciudadana en los procesos de apropiación social del conocimiento y de diseño tecnológico, financiado por el Ministerio de Economía y Competitividad de la Administración General del Estado (CSO2014-59136-P).

2 Universidad Autónoma de Barcelona (España)

E-mail: margavris@gmail.com

3 Universidad Autónoma de Barcelona (España)

E-mail: franciscojavier.tirado@uab.cat 
Keywords: biopolitics; digital humanities; global health.

Sumario: 1. Introducción. 2. Humanidades digitales y ontología en los modelos epidemiológicos: entre la ciencia y el entretenimiento. 3. Heterotopías como diseños (futuros) para el bios. 4. Nota metodológica. 5. El diseño de lo vivo; 6. Conclusiones; 7. Referencias.

Cómo citar: Gavris, M.I.; Tirado, F.J. "Recordando el futuro, imaginando el pasado. La creación de escenarios como ejercicio ontológico": Teknokultura, vol. 15 (1) 2018, pp. 23-38.

\section{Introducción}

Durante la última década, en el proceso de construcción - tanto conceptual como biopolítico — que se ha realizado sobre la "bioseguridad" (Caduff, 2008; CastilloSalgado, 2010; Hill-Cawthorne y Sorrell, 2016; Rose, 2006) —vista como espacio donde se demarca la vida - se recalca con fuerza que la propuesta de la digitalización en todos sus procedimientos, propone en última instancia conceptualizar la vida, a partir de procesos definidos por datos cuantitativos, matrices de codificación, representación y bases de datos. Según remarca la literatura especializada, estaríamos ante una mutación que lleva hacia una nueva ontología de lo vivo.

Aunque pueda parecer que los modelos tecnológicos mencionados son antagónicos con los que aparecen en las lecturas clásicas del ejercicio biopolítico (Giorgi y Rodríguez, 2007; Foucault, 2009), la digitalización vista desde el diseño de datos (por ej. minería de datos) o creación de escenarios (propuestas de respuestas frente a una crisis, proyectados en unas temporalidades futuras ambiguas), se plantean como herramientas que proponen visiones complejas sobre unos espacios heterotópicos (Foucault, 1984; Toro, 2008), donde aparecen reconfiguraciones de flujos y relaciones híbridas (Seguel y Gavris, 2015), que apuntan en la misma dirección conceptual que las mencionadas lecturas clásicas. Por ello, en este nuevo ámbito de la epidemiología se exhiben importantes desafíos para el análisis de su impacto social, de cómo está ofreciendo nuevas formas de lectura (por ej. data-research) y de cómo y por qué redefine los enfoques biomédicos (Barker, 2010; Bauer y Olsén, 2009).

En respuesta a este desafío, observamos una nueva línea de análisis que algunos autores denominan biopolítica 2.0 (Gabrys, 2014; Lacy, 2008) y que se plantea como nueva tendencia para abordar la complejidad del contexto social que se centra en una salud definida como global y como medida de control y vigilancia en una constante aceleración de los procesos de mundialización. Esto ha implicado que en la última década se hayan definido nuevas escalas de análisis, medidas de gestión del futuro (Caduff, 2008), donde la activación de nuevos procedimientos y actores, a su vez, han provocado la aparición de articulaciones que no se preveían al digitalizar los contenidos biomédicos clásicos analógicos.

La articulación de estos nuevos espacios permite preguntar si estamos frente a una nueva ontología referida a lo biótico. Una en la que los modelos tecnocráticos de diseño de la acción, como por ejemplo, el scenario-planning (escenarios) (Amer, Daim y Jetter, 2013; Kahn y Wiener, 1967; Schwartz, 1991) y el conocimiento producido por disciplinas como la "epidemiología digital" (ED); se plantean como realidades eficientes y medibles que permiten facilitar la visualización y los posicionamientos expertos para tomar decisiones en escalas diferenciadas y globales, 
utilizando rutinas de datos cuantitativos digitalizados y automatizados (por ej. big data, inteligencia artificial) que hasta el momento no eran especialmente relevantes en la definición de la mencionada realidad.

Desde el marco conceptual de los estudios de ciencia y tecnología, especialmente de los centrados en el análisis de la epidemiología (Bauer y Olsén, 2009; Tirado y Cañada, 2011; Tirado et al., 2014), se ofrece una interesante oportunidad para analizar todo esto. Consideramos tal análisis un ejercicio novedoso que apunta hacia espacios de reflexión en los que ciencias sociales y ciencias biomédicas pueden encontrarse y aunar esfuerzos. Así, en la primera parte del texto desarrollamos nuestro marco conceptual, destacando el papel de la tecnología como agente de traducción y mediador que forma parte inherente del mismo proceso. Su articulación se convierte en el modus operandi que permite entender la delimitación de la ED como una nueva "ontología inteligente" (Collier, 2012; Ferreira, Paolotti, Couto y Silva, 2013) en la salud global.

A continuación, en el material empírico que se presenta se analizan tres aspectos clave en la negociación de la definición de la mencionada ontología: a) la conformación de lo biótico como meros datos matriciales; b) la aparición de las denominadas heterotopías en salud donde se mezclan y redefinen lo vital y lo virtual; y c) la llegada de sujetos y subjetividades híbridas. Presentaremos algunos de los hallazgos empíricos de nuestra investigación utilizando el concepto escenario en nuestro análisis, como planteamiento heterótopico aplicado en salud global, que podemos rastrear a través de las herramientas de visibilización de la ED, que actúan como nuevos mediadores que sorprenden las mutaciones de su traducción de lo social. Finalmente, concluimos proponiendo que este nuevo enfoque permite activar ciertas alarmas sobre la mutación que el significado de la vida adquiere en la realidad e inteligencia que configuran estos nuevos saberes y prácticas.

\section{Humanidades digitales y ontología en los modelos epidemiológicos: entre la ciencia y el entretenimiento}

En las últimas décadas, la epidemiología ha sido objeto de un intenso análisis social y cultural. Un ámbito tan tecnificado y especializado como el que delimita esa disciplina se había mantenido durante muchas décadas protegido o al margen de su abordaje desde un punto de vista sociocultural (Seguel y Gavris, 2015; Tirado y Cañada, 2011; Tirado, et al., 2015). Esa tendencia se ha roto recientemente y han proliferado los estudios que muestran cómo la epidemiología es una potente constructora de realidad. Uno de los ámbitos en los que se ha dado este giro es el de las humanidades digitales.

La última década ha conocido el florecimiento de esta disciplina, que opera en la intersección que crean las nuevas formas de interacción, la constitución tecnológica de actores desconocidos hasta el momento y la producción de nuevos contenidos. Joseph Raben menciona por primera vez este concepto en lo que era ya una proyección de un escenario a largo plazo sobre la transformación social que producirían las tecnologías de comunicación e información, en la conformación de las "humanidades digitales", que permitiría incluir "todas las fases de las ciencias sociales que hacen hincapié en lo humano" (Prospect, 1966, p.1)

La novedad del enfoque reside en aproximarse al evento tecnológico otorgándole el estatus o valor de mediador. Es decir, de articulador y operador en la acción y pro- 
ducción de realidad. En nuestro análisis plantea que se puede extrapolar con interés al análisis de las nuevas disciplinas biomédicas. Y esto por dos razones. En primer lugar, permite caracterizar de otra manera el proceder y objetivo de las mismas. En segundo, introduce elementos de valoración ética y justicia social en su actividad. Un ejemplo privilegiado de lo mencionado es el caso que nos interesa, el de la epidemiología.

Destacamos que tradicionalmente, en la epidemiología clásica se ha operado con tres grandes dimensiones: a) la circulación acelerada de vectores infecciosos; b) la capacidad de transmisión de vectores nuevos o emergentes, y c) la evaluación de la viabilidad y eficacia de las estrategias de prevención e intervención contra pandemias o crisis epidemiológicas (Boëlle, Ansart, Cori y Valleron, 2011; Khan, Arino, Hu, Raposo, Sears, Calderon, Gardam, 2009). Planteamos que a partir de la implementación de los procesos de digitalización, aparece una mutación a nivel conceptual en relación con la nuevas formas de articulación tecno-científicas a partir de soporte técnico, sumándose una cuarta, que hace referencia a la trazabilidad de los brotes en tiempo real y la disponibilidad de sus datos en diferentes localizaciones geográficas de manera simultánea (Gavris, 2015). A partir de estas dimensiones, subrayamos que a pesar de que la epidemiología es una disciplina que se aplica con y sobre personas, que atiende a colectivos y previene fenómenos de pánico social, su enfoque es puramente técnico y médico.

Bajo este precepto consideramos que la transformación digital que ha llegado a la misma; lo que nos permite atrevernos a caracterizarla como una disciplina de las humanidades digitales porque transforma su horizonte de análisis porque:

a) Las epidemias no constituyen exclusivamente un problema biológico y médico; sino que plantean fenómenos que exhiben múltiples dimensiones, como un actor constituido socio-técnicamente que muestra diferentes dimensiones vinculadas y ensambladas por completo en una única totalidad con sentido (Tirado y Cañada, 2011).

b) Tal totalidad no resulta inocua o indiferente para el devenir de nuestra vida cotidiana, sino la transforma profundamente redefiniendo los límites, donde se borran las delimitaciones entre lo humano y lo animal, y lo que los califica como algo político o natural; porque se mezclan las diferencias entre las escalas locales y globales, y las fronteras entre lo sano y lo patológico se quiebran (Tirado, Gómez y Rocamora, 2014).

c) Pero las mencionadas rupturas no son la antesala de un estado permanente de indefinición práctica y simbólica. Todo lo contrario: constituyen el umbral para una reconstrucción posterior de los mencionados límites con otros significados y sentidos, que reconstituirán nuestras formas de vivir y pensar en lo cotidiano (Tirado, et al., 2014).

d) Pensar la nueva epidemiología como una humanidad digital abre también el camino para reconceptualizar su proceder como un espacio peculiar, como lo que Michel Foucault denominó heterotopía.

\section{Heterotopías como diseños (futuros) para el bios}

Según Foucault (1994), la noción de heterotopía se describe por tematizar una época en la que el espacio toma el relevo del tiempo en la definición de los principales 
procesos que caracterizan nuestra realidad (Foucault, 1999). En nuestro caso, nos permite crear el marco para conceptualizar las transformaciones que suponen las prácticas y discursos de la ED. Y esto ha sido así porque lo digital da voz a "otros espacios" (Gavris, Seebach, Torrejón Cano, y Tirado, 2016), a un espacio en un espacio, que es precisamente la definición de heterotopía. Así, su aplicación al ámbito de la salud permite mostrar cómo el "análisis de los espacios otros constituye una reflexión desde espacios de exclusión donde se efectúan prácticas específicas que buscan moldear al sujeto moderno como resultado del poder" (Toro, 2008, p. 7-8). Sin embargo, remarcamos que el mismo Foucault, plantea la heterotopía (1999), como línea de fuga, como un espacio que contradice esas condiciones de poder y que nos permite analizar críticamente la historia, creando espacios estéticos de resistencia y miradas alternativas (por ej. las fiestas populares, el circo, etc.).

Dentro de este marco proponemos dos tipos - complementarios - de tecnologías para observar cómo se articulan las heterotopías emergentes, como los nuevos diseños futuros del bios que aparecen en la nueva epidemiología. Por una parte, tenemos los scenario-planning (o escenarios) como herramienta de estrategia política; que se proponen como espacios de diseño donde se articulan conceptos, actores posibles y estrategias que se proyectan siempre en un espacio futuro. Por otra, la permanente digitalización epidemiológica como marco tecnológico híbrido, que cruza lo virtual y lo real en líneas de articulaciones radiales (por puntos de activación) en la gestión conocimientos que proyectan conceptos y estrategias cercanas y próximas a la ciudadanía.

\subsection{Transposición digital de los escenarios de salud pública global}

El concepto de scenario-planning ya tiene un complejo historial de varias décadas, en las cuales hay una amplia variedad de definiciones y aplicaciones (Amer, et al., 2013). Una de las pioneras (Kahn y Wiener, 1967) definió los escenarios como un "intento de describir con algún detalle una secuencia hipotética de acontecimientos que podría conducir plausiblemente a la situación prevista” (p. 262). Además, considerando los escenarios como herramientas para definir una multiplicidad de posibles futuros alternativos (Jetter, 2003); se sostiene que son "historias cuidadosamente construidas sobre el futuro destinadas a ayudar a la gente a tomar mejores decisiones frente a la incertidumbre" (Lempert, 2007, p. 110)

Sea como fuere, un escenario es mucho más que una ficción. Como señalan Van Doorn y Van Vught (1983), los escenarios son propuestas de articulación tan complejas y con tantas variantes que su implementación no puede darse al margen del soporte tecnológico. La articulación en relato y tecnología, provee sentido y oficio al primero, que no se desarrollará sin la aparición y mediación del segundo.

Esta combinación adquiere una especial relevancia en las ciencias de la vida, adonde los escenarios han sustituido a los viejos árboles de cálculo de riesgo y medida de probabilidades en la producción de conocimiento, y se han convertido en la matriz epistémica de prácticas y discursos. Existen dos enfoques principales en el diseño y la implementación de los escenarios en estas disciplinas: a) centrado en el análisis a partir de poblaciones de datos (por ej. Big Data, minería de datos) y b) 
centrado en la afectación individual, donde el mismo individuo se convierte en un generador de datos de primera mano (por ej. Quantified Self).

\subsection{Digitalización epidemiológica}

La digitalización en la epidemiología ha sido un proceso constante e imparable en las últimas décadas. Tantos sus procedimientos como sus saberes se han comenzado a articular a partir de las herramientas que ofrecen las tecnologías de la información y la digitalización: desde dispositivos para fotografías tradicionales a aplicación de herramientas informáticas (bases de datos, imágenes e impresoras $3 \mathrm{D}$, etc.) donde aparece una recogida de datos en tiempo real, acentuada por el despliegue de apps en dispositivos portátiles (al alcance de expertos y no-expertos). Para algunos autores (Rudnicki, 2017; Gavris, et al., 2016) esto supone la aparición de una nueva articulación de conocimientos y prácticas médico-epidemiológicas en diferentes escalas, utilizando procesos de afectación y participación de los ciudadanos. A todo este conjunto se le ha denominado epidemiología digital (D’Ambrosio, Tozzi y Gesualdo, 2016; Salathé, Bengtsson, Bodnar, Brewer, Brownstein, Buckee, Vespignan 2012), planteando que la vigilancia se convierte en una síntesis de temporalidades que permiten trazar líneas para permitir una articulación conceptual dinámica; donde el conocimiento que se articula se aplica en la gestión de las emergencias biomédicas, donde se intenta resolver lagunas de los modelos tradicionales de vigilancia (por ej. discrepancias de períodos de tiempo entre la detección de una emergencia y su actuación).

Conviene puntualizar la diferencia entre el concepto de epidemiología digital (o $E D)$ y telemedicina o la e-epidemiología. La telemedicina exhibe una lógica similar a la lógica lineal de diseminación de contenidos que presenta la epidemiología clásica; es de los primeros modelos de digitalización de contenidos biomédicos y epidemiológicos, pero no permite la interacción entre usuarios, ni posibilita la adaptación de contenidos. Por otro lado, la e-epidemiología (en algunos autores epidemiología 2.0) se centra más en la base técnica y tecnológica que en el impacto y el uso que el usuario final da al contenido (Brownstein, Freifeld y Madoff, 2009); funciona como transcripción técnica de las categorías utilizadas posteriormente para la codificación semántica (Ekman y Litton, 2007).

Por el contrario, la epidemiología digital (Salathé et al., 2012; Vayena et al., $2015)$ proporciona una visión holística que tiene impacto hasta en la misma conceptualización de las prácticas de riesgo en el campo de la salud global (más allá las simples partes técnicas). En la ED, las conexiones se activan a través de los sujetos que se conectan como complementos tecnológicos (Latour, 1991, 2005), permitiendo la visualización y el movimiento acelerado de la información "para identificar patrones espacio-temporales en los que se necesita con urgencia el conocimiento de los factores impulsores de la dinámica de la enfermedad" (Bakker, Martinez-Bakker, Helm y Stevenson, 2016, p. 6693).

No obstante, las caracterizaciones habituales de la ED soslayan que todo el aparato técnico de la misma opera gracias al uso intensivo de escenarios. Por tanto, ambos conceptos no se pueden desligar, porque en nuestra opinión son las fuentes de heterotopías que mencionábamos previamente que ambos son responsables del diseño de un futuro que afecta al presente, redefiniendo en sus articulaciones al mismo concepto de vida. 


\subsection{El diseño del futuro acercándose}

Los nuevos enfoques para las herramientas digitales de detección de enfermedades están planteando la necesidad de repensar la vigilancia desde un espacio denominado Salud Pública Global. La ED contribuye activamente a ese objetivo y lo hace ofreciendo espacios de encuentro (teóricos y empíricos) en los que la aplicación en formatos tecnológicos de los escenarios de referencia (por. ej. proyección temporal por décadas de modelos de ciudadanía y salud pública), proponen un campo de investigación relativamente nuevo y que aparece en la última década como una delimitación conceptual interdisciplinaria. Además, la ED cuestiona cómo se crean los filtros (ref. técnica informática) utilizados en el diseño de los contenidos de las políticas de salud global, que según se destaca en la literatura abre "el acceso a nuevas fuentes de datos y permite extraer significado de información no estructurada y compleja" (D’Ambrosio, Tozzi y Gesualdo, 2016, p.37).

Planteamos que la principal novedad radica en la articulación transversal de los elementos interconectados, que afectan múltiples niveles del proceso de evaluación e intervención en caso de emergencia (Gavris, et al., 2016). Nos referimos, por ejemplo a la mutación conceptual y performativa que plantean las nuevas plataformas tecnológicas suponen (HealthMap, BioCaster, EpiSPIDER, Twitter, etc.), permiten que la interacción entre elementos biomédicos o sociales o políticos; que muten desde articularse en base a una lógica lineal clásica de gestión de datos a una lógica de comunicación radial y dinámica (se (re)construyen por puntos de activación temporal y aleatorio) (Burt, 1992).

Este nuevo enfoque subraya la articulación radial de los nuevos mediadores, donde identificamos nuevas agencias que permiten aparecer espacios de traducción y mediación. Por ejemplo, los gestores de datos (responsables técnicos de diseño contenidos web), cobran importancia en el ámbito digital, potenciando formas alternativas en plantear nuevos espacios, que permiten que surjan nuevas voces y la articulación de nuevas habilidades que reconfiguran el contexto.

La ED acerca los escenarios que usan técnicos y especialistas para crear conocimiento epistémico válido a los ciudadanos. Lo hace a través del enorme dispositivo tecnológico que suponen las apps, programas de computación, juegos, etc. Y lo hace con la finalidad última de integrar a este en un proceso de vigilancia que sería constante y perpetuo. La opción de registrar en una aplicación de móvil datos sobre enfermedades, síntomas o señales, que se reflejan inmediatamente en un mapa interactivo (por ej. Healthmap, Google Flu) es una realidad que reconfigura nuestra concepción de la vigilancia epidemiológica. Planteamos que se convierte en una observación constante de uno mismo y de su entorno y ofrece un registro de datos que permite la trazabilidad de datos a posteriori. A continuación en nuestro análisis, presentamos varios ejemplos de escenarios para observar las implicaciones que plantean estos nuevos conjuntos de prácticas y saberes.

\section{Nota metodológica}

El presente análisis se plantea como parte de una investigación sobre epidemias y biopolítica que se está desarrollando (desde 2013) en el marco del grupo de Estudios de Ciencia y Tecnología de Barcelona (STS-b); donde se realizó un análisis 
de modelos epidemiológicos actuales y las nuevas tendencias en el manejo de los datos en salud global, para aclarar cuestiones relacionadas con la bioseguridad y la gestión de emergencias biomédicas. Para este fin se ha desarrollado un diagrama de análisis para poder triangular información de fuentes de distintos formatos, para observar tendencias en la última década sobre: a) tipos de sujetos que se mencionan; b) técnicas y tecnologías referentes y c) las tramas de significación que se entrelazan; con especial enfoque en las mutaciones que plantean. Respectivamente se han utilizado los siguientes datos empíricos: a) escenarios e informes de diversas instituciones; b) 15 grupos focales heterogéneos; c) marcos normativos que ubican mutaciones en epidemiología desde el ángulo de lo digital; d) entrevistas en profundidad y e) materiales variados con soporte digital (por ej. artículos periodísticos).

El análisis propuesto en el presente artículo, se plantea a partir de la triangulación de diferentes fuentes: a) escenarios y artículos periodísticos ${ }^{4}$ vinculados epidemiología, b) entrevistas personales ${ }^{5}$ y c) grupos focales ${ }^{6}$; para observar cómo se articulan los mecanismos principales de la nueva ontología en salud, donde se reconfiguran las relaciones entre las políticas de salud global.

\section{El diseño de lo vivo}

Como hemos indicado previamente, hemos utilizado tres ejes de análisis: a) bios como datos; b) heterotopías en salud donde se redefine tanto lo vivo como lo virtual; y c) sujetos y sus subjetividades. Para ilustrar cada eje hemos recogido el ejemplo de un escenario que remarca las transformaciones conceptuales y las prácticas que han aparecido; ampliando en base a tres subcategorías.

\subsection{Bios como datos}

Bios, concepto de origen griego, plantea dos definiciones. Por una parte, puede ser entendida como enfoque sobre la vida; $y$, por otra parte, entendida como BIOS (sistema de arranque de los sistemas informáticos) que permiten la articulación de los planteamientos sobre la vida. El mismo concepto de ED los engloba a ambos, pero también exige atención sobre la transformación conceptual de las amenazas biológicas. Nuestro análisis se inicia a partir de un escenario que hace referencia al uso de los datos científicos vs. la ciencia ciudadana; que permite observar a posteriori tres subcategorías que definen el nuevo despliegue de planteamientos sobre la vida a través de la implementación de modelos tecnológicos:

[...] hace referencia a una base de datos vinculada a miles de proyectos científicos de la ciudad [que comparten] sus datos con el repositorio de datos más apropiado,

\footnotetext{
Análisis de contenido de materiales variados con soporte digital: plataformas digitales y artículos periodísticos. Desarrollados entre 2013-2015, con 1 epidemiólogo, 1 veterinario y 2 gestores de datos biomédicos plataformas digitales

6 Desarrollados entre 2014-2015, en varias ciudades españolas, con expertos en gestión de datos, investigadores sociales, veterinarios, estudiantes universitarios, periodistas, grupos de activistas y feministas, etc.
} 
exportan los datos locales según sea necesario e incluso expanden sus esfuerzos para incluir el monitoreo de la biodiversidad (EPA ${ }^{7}, 2016$, p. 12)

En primer lugar, aparece una mutación en la (bio)política de los acontecimientos (por ej. protesta en defensa de la enfermera infectada de Ébola), replanteando cómo se articulan los contenidos y las vivencias diarias entorno a eventos especiales, cómo aparecen nuevas interacciones con el mismo contenido epidemiológico y la escalada para la defensa de estos en manifestaciones y redes sociales. En el caso de enfermera infectada en España durante la epidemia de Ébola en 2014, se remarca la articulación de contenidos dispares (el perro, los vecinos, el ascensor, etc.) para observar la discrepancia vivencial de los vecinos de la enfermera, que una vez diagnosticada fue ingresada:

[...] no salen de su asombro cuando El Confidencial les confirma que su vecina y su esposo están aislados [...] 24 horas después de que estallara la crisis de la enfermedad en España, nadie ha acudido al epicentro de la epidemia para informar sobre lo sucedido (García, 2014, s.p.)

En segundo lugar, cuestionamos si aparece una redefinición del concepto humanidad a través de los nuevos planteamientos sobre lazos sociales y los estatus políticos planteados sobre todo en las plataformas digitales (amigo, ciudadano, comunidad); en la definición de las humanidades digitales ${ }^{8}$ como espacios donde la "co-creación [implica] el trabajo en equipo, los roles especializados dentro de los equipos y los estándares de "producción" que implican la especialización se convierten en características definitorias del giro digital en las ciencias humanas".

En último lugar, la promoción de la (auto)vigilancia de la salud nos transforma en un nuevo cyborg que genera datos, se (auto)cuantifica y está procesada en flujos de datos ajenos a los matices cualitativos del contexto social donde estos se generaron (por ej. movimiento quantified self $)^{\text {) }}$; donde el bios y nosotros mismos nos transformamos en un algoritmo:

La gran diferencia de esta pandemia, a comparación con las otras pandemias anteriores, es el componente tecnológico. ¡Creo que la gran diferencia fue que era una pandemia monitorizada en tiempo real! Anteriormente en las pandemias no podías tener la información en tiempo real (entrevista veterinario; 2013)

Como se desprende de todo lo anterior, lo biótico carece de componentes fenomenológicos e integrales. Es un despliegue de datos que se pueden fragmentar, recomponer, analizar y desterritorializar de los espacios concretos en los que despliega su actividad.

\footnotetext{
Environmental Protection Belongs to the Public - A Vision for Citizen Science at EPA https://www.epa.gov/ sites/production/files/2016-12/documents/nacept_cs_report_final_508_0.pdf

$8 \mathrm{http}: / /$ www.humanitiesblast.com/manifesto/Manifesto_V2.pdf

9 http://quantifiedself.com/
} 


\subsection{Heterotopías en salud}

Cuestionamos cómo se despliegan a partir de un escenario, las lecturas que plantea para la rearticulación de los elementos dispares a través de la mediación tecnológica, donde se transmite una demarcación futura de la humanidad y los profesionales sociales. Para este fin proponemos el siguiente fragmento de un escenario, que plantea una innovación tecnológica de la atención virtual de salud mental:

Todos los servicios de salud mental, con la posible excepción de la atención de emergencia, serían proporcionados en línea y por profesionales virtuales de la salud mental. El profesional virtual no sería un ser humano, sino un avatar con inteligencia artificial [...] Algunos pacientes todavía pueden querer algún contacto con seres humanos reales para supervisar o validar sus interacciones con los clínicos virtuales [...] Los facilitadores humanos podrían ayudar a los pacientes a navegar por el software (Giacco, Amering, Bird, Craig, Ducci, Gallinat, Priebe, 2016, p.3)

$\mathrm{Su}$ lectura nos abre un abanico de preguntas sobre el futuro de la medicina personalizada: ¿qué aspectos de salud se delimitan? ¿Por qué plantea la transversalidad del mismo paciente que requiere atención sine qua non y en cualquier momento? ¿Los facilitadores humanos serán médicos, psicólogos o gestores de contenidos?

En primer lugar, planteamos que la relación entre lo biótico y lo virtual se redefine en los espacios heterotópicos que abre la ED. Esa mutación es muy clara en el debate sobre los desafío y procesos que (se) plantean los diseñadores de inteligencia artificial (IA), que requiere que los makers (técnicos que articulan desde nivel conceptual hasta su puesta en práctica) tengan soporte de las ciencias sociales hacia una visión crítica sobre la implementación de los procesos tecnológicos y las rupturas que podría provocar a medio-largo plazo donde la "investigación en tecnología suele ser impulsada por datos duros. Sin embargo, los seres humanos son impredecibles, y cuando están construyendo la IA, se enfrentan con la imprevisibilidad adicional de un espacio desconocido" (EPIC 2017 ${ }^{10}$ )

Los escenarios aplicados marcan vías de posicionamiento para los expertos, marcando desde el mismo diseño tecnológico una mutación que se plantea al propio uso propuesto para las plataformas, formas de articulación de contenidos y agentes implicados (escalas y temporalidades). Estos, a posteriori son rastreables gracias a la inscripción de datos virtuales que son fácilmente ubicables indiferente de la localización y franja temporal en la que se generaron. Por ejemplo, en una de las entrevistas sobre la gripe porcina 2009, se menciona que: "hacerlo a través de la comunidad virtual permite explorar una posibilidad, un escenario (entrevista médico epidemiólogo; 2013)

En segundo lugar, se destaca que está surgiendo en los últimos años una nueva estética cinematográfica digital (por ej. explosión de imágenes, infografías y documentales (no)expertos sobre temas epidemiológicos); donde por ejemplo los proyectos se convierten en apps para: "incentivar la ciudadanía geográfica [es clave] para capturar y analizar la información recopilada por parte de los ciudadanos y los científicos" (EURECAT, 2017, s.p.)

10 https://www.epicpeople.org/people-who-build-with-ai/ 
Por último, los límites de la experiencia se renegocian en proyecciones futuras de escenarios plantean saltos de y entre escalas, en el despliegue de estrategias de salud pública local o global. Proponemos como ejemplo el cuestionamiento sobre las definición de la salud, en base a tres escenarios post-Brexit y sus respectivas implicaciones donde "el impacto potencial en la salud y la atención sanitaria será relevante [...] mientras que la administración pública parece estar luchando para hacer frente, especialmente dentro del Departamento de Salud, que ha experimentado redundancias a gran escala (Fahy, et al., 2017, p.1). Además, se remarca la actuación como mediados de la tecnología, que posibilita saltos entre escalas (tipos de conocimiento o agentes implicados), donde "los ciudadanos no-expertos se han convertido en expertos a base de hacer preguntas y aprender. La tecnología facilita todo esto a una escala global" (entrevista médico epidemiólogo; 2013)

Espacios dentro de espacios, que plantean la dinámica de acción de la ED, donde se negocian y construyen muchos elementos; como por ejemplo, las maneras de mirar y leer la información, de acercarnos a un fenómeno y de entender cómo se plantea que es un sujeto.

\subsection{Sujetos y subjetividades}

Planteamos la reflexión sobre cómo se planea moldear sujetos según la ED, a partir de una propuesta normativa problemática, propuesta como puente entre dos horizontes conceptuales, hacia un horizonte donde los usuarios podrían decidir sobre sus propios datos que los definen. Nos referimos a Ley de Derecho al Olvido, propuesta en 2012 por la Unión Europea, que opera como un excelente escenario para resaltar los desafíos que plantea la ED en términos de definición de nuevos sujetos de acción y otras subjetividades, su implementación en la salud, su respectiva implicación en la gestión de contenidos y su aplicación en la práctica. Plantemos para nuestro análisis un fragmento de un escenario EPIC (Centre of Expertise on Animal Disease Outbreakes), para traer en el debate los interrogantes que está ley plantea para el futuro de la salud global. Por ejemplo, según el siguiente caso, bajo el amparo de esta ley podríamos encontrar "información sesgada" sobre históricos personales o contextuales de datos epidemiológicos, donde "la nueva legislación para la protección de los datos para su uso en el futuro $[. .$.$] tiene importantes implicaciones para los estudios de vigilancia y epidemio-$ lógicos" y "requiere consentimiento activo para el uso de datos" (EPIC, 2014, s.p.)

En primer lugar conviene señalar que la digitalización permite la aparición de nuevas formas de (definir la) participación en emergencias biomédicas. Proponemos como ejemplo el análisis sobre los nuevos elementos de referencia para definir a los pacientes:

ya que los individuos están empezando a medir, rastrear, experimentar, intervenir, tratar e investigar sus condiciones y síntomas, genomas, biomarcadores, comportamiento y medio ambiente, individualmente y en colaboración con otros [que] surgen del análisis de modelos emergentes de atención de salud guiados por los pacientes (Swan, 2009, p. 521)

En segundo lugar debe mencionarse que en los últimos años ha aparecido un intenso debate en la literatura sobre la redefinición de del sujeto, en debates como los 
sobre bienes públicos, ciencia y difusión del conocimiento especialmente desde los movimientos sociales asociados a las nuevas tecnologías de información y conocimiento (TIC):

lo que hablamos ahora es muy importante, porque hay una convergencia entre tecnología...big data[...] va a magnificar todo esto en una dimensión que quizás no nos esperamos [...] hace 15 años no nos imaginábamos que pasaría con esto del internet [...] esa incapacidad de visualizar...de la mayoría...la magnitud del problema [...] pero también toda la capacitación y empoderamiento del ciudadano puede jugar a nuestro favor (grupo focal activistas en movimientos sociales; 2016)

Por último, consideramos importante señalar los cambios que plantea la afectación directa de la ciudadanía. Por ejemplo, la reacción frente a una crisis sanitaria global de la pandemia 2009, cuando "durante este periodo sentía la necesidad de participar en estas comunidades, pero al mismo tiempo sentía la responsabilidad a nivel profesional" (entrevista medico epidemiólogo; 2013).

Si añadimos las nuevas concepciones sobre colectivos (según las interacciones que plantean) y respectivamente las disrupciones que plantean (Latour, 2005), planteamos que la ED no es ajena a la construcción de sujetos y subjetividades. Su implementación ofrece un nuevo tipo de sujeto, activo, implicado en una observación permanente de tipo vigilante frente a las amenazas biológicas y una subjetividad vinculada al riesgo y a un tipo de amenaza ubicada en el futuro y con una posible escala global.

\subsection{Para recordar el futuro...}

En los últimos años, diversos trabajos han coincidido en señalar que las propuestas foucaultianas sobre el biopoder necesitan ser revisadas. Entre los diversos argumentos que se han presentado para tal reformulación, destaca el que se centra en la emergencia de las ciencias biomédicas y la reconceptualización que suponen de la noción de vida. Nosotros, sin embargo, hemos planteado que esa no es la única transformación que el concepto está sufriendo en nuestro presente. Dispositivos como la ED realizan un ejercicio similar. La única diferencia con el planteado por autores como Nikolas Rose (2006) es que la vida no se moleculariza, sino que se convierte en una trayectoria, que se despliega articulando distintas escalas y que es, fundamentalmente, algo representable a través de mapas, diagramas, etc. Es decir, emerge una nueva ontología de lo vivo.

\section{Conclusiones}

Al referirnos a ejercicios ontológicos de lo vivo, que plantean los escenarios en la epidemiología digital, hemos remarcado en el análisis del presente artículo que los escenarios actúan como matriz epistémica de prácticas y discursos, marcando ya hace décadas las direcciones de implementación de los planteamientos conceptuales. La capacitación de estas articulaciones fue potenciada por la tecnología, que actúa como agente de traducción y mediador inherente del mismo proceso, planteando la ED como una nueva ontología inteligente (Collier, 2012; Ferreira, et al., 2013) 
en la salud global. Donde los nuevos espacios, más allá de los meros desplegables técnicos, provocan profundas mutaciones conceptuales, porque - sobre casos como pandemia 2009 - "toda la información que se ha recogido [...] dará muchísima información a los científicos para poder valorar qué ha pasado y todo lo que ha pasado con las redes sociales...como se han activado las redes sociales durante esta" (entrevista veterinario; 2013)

La mencionada nueva ontología de la vida que emerge en la ED tiene las siguientes características, según los ejes de análisis que hemos propuesto en la primera parte.

En primer lugar, como visión global, planteamos que la noción de vida adquiere su pleno significado en el interior de dos conjuntos diferentes de elementos: a) sólo puede ser aprehendida e identificada gracias a protocolos de seguridad, dispositivos de vigilancia y rastreo, datos estadísticos que se elaboran en centros de vigilancia epidemiológica, imágenes que representan vectores infecciosos, pruebas médicas, etc.; y b) su definición se torna plena cuando se la vincula al movimiento de la información y su representación en bases de datos y gráficos. Es decir, la ED rompe con la imagen vitalista decimonónica del sentido común que muestra lo viviente como una potencia que está más allá del alcance de cualquier regulación o sistematización completa, como un conjunto de elementos materiales, normas y regulaciones. Si hay código se puede hablar de vida, si no, entramos en el terreno de "lo indefinible".

En segundo lugar, referente al bios transformado en un algoritmo, remarcamos que lo biótico se torna algo controvertido, en la medida en que se constituye como una permanente amenaza, donde la vida se vigila por ser fuente de riesgo, donde se abre un espacio en el futuro en el que se conforma una amenaza perpetua; donde: "no sabemos qué pasará, pero sabemos que tarde o temprano pasará algo grave debido al contagio entre especies" (entrevista veterinario; 2013).

En tercer lugar, heterotopías en salud donde se redefine tanto lo vivo como lo virtual. La ED pone en igualdad los elementos y las escalas, que hasta el momento estaban completamente separados. Articulan en un mismo tejido: política y biología, conocimiento experto y lego, entidades nacionales e internacionales, sistemas de vigilancia muy sofisticados con prácticas agrarias milenarias...

Por último, referente a los sujetos híbridos, donde la vida entendida a partir de su vínculo inter-especie moviliza en un mismo plano distintas instituciones y niveles de acción; donde la representación como trayectoria establece vectores que cruzan y aúnan en totalidades comunes todas esas diferentes instancias y escalas. Probablemente la mayor expresión de esta puesta en igualdad la constituya el conjunto de expresiones One Health, Global Health o One World One Health que recogen el espíritu de un movimiento interdisciplinar que aboga por el desarrollo de unas ciencias médico-biológicas que atiendan la necesidad de un bienestar global que no establezca diferencias ontológicas definitivas entre seres humanos, animales y medio ambiente.

La ED constituye un dispositivo discursivo y práctico que no se limita a redefinir la noción de seguridad o vigilancia. Plantear una lectura sobre los escenarios usados en ED, como noción de frontera (Latour, 1987), no hace referencia a una línea divisoria, sino se subraya que lo biótico en nuestra última gran frontera. Sin embargo, sus límites van mucho más allá de los de la política tradicional y conforman un verdadero proyecto biopolítico. La vida es permanente relación e intercambio de información y por tanto un límite constante. Lo vivo se extiende por todos los rincones 
de nuestro entorno y transforma el planeta en un gigantesco espacio liminal. Pero ¿limítrofe con qué?

\section{Referencias}

Amer, M., Daim, T. U., y Jetter, A. (2013). A review of scenario planning. Futures. http://doi. org/10.1016/j.futures.2012.10.003

Bakker, K. M., Martinez-Bakker, M. E., Helm, B., y Stevenson, T. J. (2016). Digital epidemiology reveals global childhood disease seasonality and the effects of immunization. Proceedings of the National Academy of Sciences, 113(24), 6689-6694. http://doi.org/10.1073/pnas.1523941113

Barker, K. (2010). Biosecure citizenship: Politicising symbiotic associations and the construction of biological threat. Transactions of the Institute of British Geographers, 35(3), 350-363. http://doi.org/10.1111/j.1475-5661.2010.00386.x

Bauer, S., y Olsén, J. E. (2009). Observing the others, watching over oneself: Themes of medical surveillance in society. Surveillance and Society, 6(2), 116-127.

Boëlle, P. Y., Ansart, S., Cori, A., y Valleron, A. J. (2011). Transmission parameters of the A/H1N1 (2009) influenza virus pandemic: A review. Influenza and Other Respiratory Viruses. http://doi.org/10.1111/j.1750-2659.2011.00234.X

Brownstein, J. S., Freifeld, C. C., y Madoff, L. C. (2009). Digital disease detection-harnessing the Web for public health surveillance. New England Journal of Medicine, 360, 2153 2157. http://doi.org/10.1056/NEJMp1002530

Burt, R. S. (1992). Structural Holes. En: The Social Structure of Competition (pp. 8-49). http://doi.org/10.1016/j.jet.2007.01.006

Caduff, C. (2008). Anticipations of Biosecurity. En: Lakoff, A. y Collier, S. (eds.) Biosecurity Interventions: Global Health and Security in Question. Columbia University Press: New York, pp. 257-277.

Castillo-Salgado, C. (2010). Trends and Directions of Global Public Health Surveillance. Epidemiologic Reviews, 32(1), 93-109. http://doi.org/10.1093/epirev/mxq008

Collier, N. (2012), Uncovering text mining: a survey of current work on Web-based epidemic intelligence Global Public Health: doi: 10.1080/17441692.2012.699975.

D’Ambrosio, A., Tozzi, A. y Gesualdo F. (2016.) Digital epidemiology. Using the internet for population health. How to listen and what can we discover: Angelo D'Ambrosio, European Journal of Public Health, Volume 26, Issue suppl_1, 1 November 2016, ckw164.081, https://doi.org/10.1093/eurpub/ckw164.081

Ekman, A., y Litton, J. E. (2007). New times, new needs; E-epidemiology. European Journal of Epidemiology. http://doi.org/10.1007/s10654-007-9119-0

EPIC Advancing the Values of Ethnography in Industry (2017) 'A Researcher's Perspective on People Who Build with AI'; (Ellen Kollstro, Sept. 2017), [en línea]. https://www. epicpeople.org/people-who-build-with-ai/, [2018, 22 de febrero].

EPIC Centre of Expertise on Animal Disease Outbreakes (2014) Scenario-planning, [en línea]. http://www.epicscotland.org/our-research/encouraging-interdisciplinarity/scenarioplanning/scenario-planning-the-future-of-animal-health-surveillance-in-scotland/driversof-change/society/, [2018, 22 de febrero].

EURECAT (2017) Fem ciencia ciudadanía a través del móvil EURECAT. Centre Tecnològic de Catalunya (juny 2017), [en línea]. https://eurecat.org/fem-ciencia-ciutadana-a-traves-del-mobil/ 
Fahy, N., Hervey, T., Greer, S., Jarman, H., Stuckler, D., Galsworthy, M., y McKee, M. (2017). How will Brexit affect health and health services in the UK? Evaluating three possible scenarios. The Lancet. http://doi.org/10.1016/S0140-6736(17)31926-8

Ferreira, J. D., Paolotti, D., Couto, F. M., \& Silva, M. J. (2013). On the usefulness of ontologies in epidemiology research and practice. Journal of Epidemiology and Community Health May 2013 Vol 67 No 5

Foucault, M. (2009). El nacimiento de la biopolítica: Curso en el Collège de France (19781979). Akal Universitaria. http://doi.org/10.1017/CBO9781107415324.004

Foucault, M. (1999). Espacios diferentes. Obras Esenciales Vol. III, Paidós. 431-441.

Foucault, M. (1994). Un diálogo sobre el poder. Barcelona: Altaya

Foucault, M. (1984). Space, Knowledge and Power; En: Paul Rabinow (ed.) The Foucault Reader London: Routledge (239-256)

Garcia,A.I., (07.10.2014) Los vecinos de la enferma de ébola: "No ha venido nadie a desinfectar el edificio" El confidencial. https://www.elconfidencial.com/espana/2014-10-07/losvecinos-de-la-enferma-de-ebola-no-ha-venido-nadie-a-desinfectar-el-edificio_230111/

Gabrys, J. (2014). Programming environments: Environmentality and citizen sensing in the smart city. Environment and Planning D: Society and Space, 32(1), 30-48. http://doi. org/10.1068/d16812

Gavris, M.I.; Seebach, S.; Torrejón Cano, P. y Tirado, F. (2016). Alternative actors y emergency warning systems. En Steward, F. (Presidencia). Science y technology by other means: Exploring collectives, spaces and futures. 4S/EASST conference. Barcelona

Gavris, M.I. (2015). Tecnociencia en contexto pandémico: revisión y discusión sobre nuevos actores. En Cubells, J., Enciso, G. y Pujol, J. (Presidencia). In Critical Social Psychology Conference: Affect, Embodiment And Politics. Barcelona

Giacco, D., Amering, M., Bird, V., Craig, T., Ducci, G., Gallinat, J., ... Priebe, S. (2017). Scenarios for the future of mental health care: a social perspective. The Lancet Psychiatry. http://doi.org/10.1016/S2215-0366(16)30219-X

Giorgi, G. y Rodríguez, F. (comps.) (2007). Ensayos sobre Biopolítica, excesos de vida. Paidós: Barcelona

HillI-Cawthorne, G.A. y Sorrell, T.C. (2016). Future directions for public health research in emerging infectious diseases. Public Health Research and Practice. 2016; 26(5):e2651655. DOI: http://dx.doi.org/10.17061/phrp2651655

Jetter A.J.M. (2003). Educating the Guess: Strategies, Concepts, and Tools for the Fuzzy Front End of Product Development, Portland International Center for Management of Engineering and Technology (PICMET), Portland: USA, 2003, pp. 261-273.

Kahn, H. y Wiener, A.J. (1967). The Year 2000: A Framework for Speculation on the Next Thirty-Three Years New York: The Hudson Institute, Inc., 1967)

Khan, K., Arino, J., Hu, W., Raposo, P., Sears, J., Calderon, F., ... Gardam, M. (2009). Spread of a Novel Influenza A (H1N1) Virus via Global Airline Transportation. New England Journal of Medicine, 361(2), 212-214. http://doi.org/10.1056/NEJMc0904559

Lacy, M. (2008). Designer Security: Control Society and MoMA's SAFE: Design Takes On Risk. Security Dialogue, 39(2-3), 333-357. http://doi.org/10.1177/0967010608088781

Latour, B. (2005). Reassembling the social: An introduction to actor-network-theory. Oxford University Press.

Latour, B. (1991). Society is Technology made durable. En: Law, J. (ed.). A Sociology of Monsters. Essays on Power, Technology and Domination. Routledge.

Latour, B. (1987). Science in Action: How to Follow Scientists and Engineers Through Society Harvard University Press. 
Lempert, R. (2007). Can Scenarios Help Policymakers Be Both Bold and Careful? En: Fukuyama, F. (ed.) Blindside: How to Anticipate Forcing Events and Wild Cards in Global Politics New York: Basic Books.

Prospect (1966). Computers and Humanities: a newsletter. I (1) p. 1-2.

Rose, N. (2006). The Politics of Life Itself: Biomedicine, Power, and Subjectivity in the Twenty-First Century. Princeton: Princeton University Press.

Rudnicki, S. (2017) The Body, Technology and Translation: Mapping the Complexity of Online Embodiment Sociological Research Online, 22 (2), 13.

Salathé, M., Bengtsson, L., Bodnar, T. J., Brewer, D. D., Brownstein, J. S., Buckee, C., ... Vespignani, A. (2012). Digital epidemiology. PLoS Computational Biology, 8(7). http:// doi.org/10.1371/journal.pcbi.1002616

Seguel, A. y Gavris, M. (2015). Tecnociencia en contexto pandémico. Centro de Estudios Epidemiológicos sobre las ITS/HIV/SIDA de Catalunya. Barcelona

Swan, M. (2009). Emerging patient-driven health care models: An examination of health social networks, consumer personalized medicine and quantified self-tracking. International Journal of Environmental Research and Public Health, 6(2), 492-525. http://doi.org/10.3390/ijerph6020492

Schwartz, P. (1991) The Art of the Long View: Planning for the Future in an Uncertain World. Doubleday, New York. USA.

Tirado, F., Gómez, A., y Rocamora, V. (2015). The global condition of epidemics: Panoramas in A (H1N1) influenza and their consequences for One World One Health programme. Social Science and Medicine, 129, 113-122. http://doi.org/10.1016/j.socscimed.2014.09.003

Tirado, F.; Baleriola, E.; Giordani, T. y Torrejón P. (2014) Subjetividad y subjetivadores en las tecnologías de bioseguridad de la unión europea Revista Polis e Psique 2014; 4(3): 23-50.

Tirado, F., y Cañada, J. A. (2011). Epidemias: Un nuevo objeto sociotécnico. Convergencia, 18(56), 133-156.

Toro, M.C. (2008). La Heterotopía En Michel Foucault Como Concepto Estético (Tesis, Universidad De La Salle Bogotá), [en línea]. http://repository.lasalle.edu.co/bitstream/ handle/10185/13499/T30.08\%20T634h.pdf?sequence=1, [2018, 22 de febrero].

Vayena, E., Salathè, M., Lawrence, M., y Brownstein, J. (2015). Ethical challenges of big data in public health. PLoS Computational Biology.

Van Doorn, J.W.M. y Van Vught, F.A. (1983). Futures research in the Netherlands 19601980, Futures 15 (1983) 504-516. 